\title{
COMUNICACIÓN INTERPRETATIVA, AMBIENTAL, CULTURAL Y ECOLOGICA
}

\section{COMMUNICATION INTERPRETATION, ENVIRONMENTAL, CULTURAL AND ECOLOGICAL}

Lic. Juana María del Consuelo Vergara Torres, MSc.

Universidad de Guayaquil, Ecuador.consuelitovtorres@hotmail.es

Lic. Ángela Yamil De Haz Cruz, MSc.

Universidad de Guayaquil, Ecuador.

Lic. María José Constante Vergara.

\section{RESUMEN}

El presente estudio se fundamenta en establecer una herramienta de comunicación que combine todos los elementos que entran dentro de un centro de interpretación ambiental, cultural y ecológico, aquí se trata de manera relevante el ámbito interpretativo combinados en primar lo artístico con lo educativo el lenguaje a veces complejo de nuestro legado cultural y natural a una forma no técnica casi coloquial y comprensible para los no entendidos en los hallazgos patrimoniales que visitan el lugar, la metodología de gestión de diseño comunicativo es crear técnicos especialmente con un tratamiento pedagógico, adecuado al nivel educativo en que se encuentran los itinerarios.

Palabras claves: interpretación, ambiental, cultural y ecológico.

\begin{abstract}
The present study is based on establishing a communication tool that combines all the elements that fall under an environmental, cultural and ecological interpretation center, here is how relevant the interpretive scope combined in sometimes complex of our cultural and natural heritage to an almost colloquial non-technical form and language understandable for non-connoisseurs in heritage finds that visit the place first the artistic with the educational management of communicative design methodology is to create technicians especially with pedagogical treatment appropriate to the level of education in that the routes are.
\end{abstract}

Keywords: interpretation, environmental, cultural and ecological.

Recibido: 29 de noviembre de 2016

Aprobado: 15 de marzo de 2017

Publicado: 30 de junio de 2017

\section{INTRODUCCIÓN}

Los centros de interpretación son equipamientos ambientales que cumplen la función de informar y orientar a los ciudadanos en sus visitas a los espacios naturales, para ofrecer un mejor conocimiento y disfrute de los valores naturales y culturales que albergan. Los centros de interpretación son una herramienta para el conocimiento y sensibilización de la población, sobre los valores naturales y culturales de los espacios donde seasientan.
La función principal de un centro de interpretación es promover un ambiente para el aprendizaje creativo, buscando revelar al público el verdadero significado natural o cultural de los bienes que expone. Se consideran además cuatro funciones básicas: Investigación, conservación, divulgación y puesta en valor del objeto que lo constituye. Se interpreta para revelar significados. Interpretar es traducir el lenguaje técnico y a veces complejo del legado natural, cultural y 
patrimonial, a una forma sencilla $y$ comprensible para el público. Interpretar puede entenderse entonces como el arte de presentar al público un lugar o un objeto, o un conjunto de ellos, para informarlo, entretenerlo y motivarlo al conocimiento. El fin de la interpretación es dejar en el visitante un entendimiento de por qué y en qué sentido es importante ese lugar y los objetos que se exponen.

Dada esta situación es necesario y estratégico contar con el espacio físico que permita aprovechar este recurso en su totalidad. Esta infraestructura deberá ser creada con una perspectiva social, cultural, natural, y económica sostenible brindando a las comunidades del sector una alternativa de desarrollo, además servirá para la preparación física y psicológica de sus educandos.

La interpretación ambiental es considerada como una actividad educativa orientada a revelar significados y relaciones mediante el uso de objetos originales, a través de experiencias de primera mano y medios ilustrativos, lejos de comunicar información literal, lo que busca es transmitir ideas y relaciones a partir de un acercamiento directo entre la audiencia y los recursos que se interpretan. Para lograrlo se utilizan diferentes técnicas que ayudan a las personas a entender y apreciar lo que se observa. La meta es comunicar un mensaje y traduce el lenguaje técnico de los profesionales en términos e ideas que las personas en general entienden fácilmente.

En estos casos se generan beneficios tanto para los visitantes como los recursos naturales y culturales en sí mismos, ya que se promueve un mayor entendimiento $y$ sensibilización de la audiencia hacia estos.

Es necesario contar con técnicas atractivas que canalicen los deseos del público de desarrollar alguna actividad relativa a nuestros programas de interpretación ya que no debemos asumir que el visitante de áreas naturales busca algo parecido a la interpretación ambiental como actividad preconcebida; por lo tanto debe emplearse algún esfuerzo para inculcar en esos deseos de actividad del público el valor de la interpretación ambiental.

Sus actividades están dirigidas a la población en general, dedicando especial atención a las visitas de grupos organizados. Un centro de interpretación desarrolla un conjunto de actividades de comunicación con el público visitante cuyo objetivo es revelar y explicar el papel y el significado del patrimonio histórico y cultural mediante su interpretación contemporánea, con el fin de aumentar la sensibilización del público y de hacer más eficaz su conservación. Debe disponer, como condición fundamental de funcionamiento, de personal especializado para la realización de los itinerarios didácticos y para la atención al público. La Interpretación puede entenderse como una herramienta de comunicación que combina tanto los elementos artísticos, creativos e imaginativos, como los técnicos, métodos, procedimientos, para transmitir un mensaje positivo y efectivo en relación con el entorno visitado, poniéndolo alcance, utilizando un lenguaje sencillo y claro, de cualquier tipo de público: local, turista, jóvenes estudiantes, mayores, etc.

\section{Características de la Interpretación Ambiental:}

a. Es amena. Mantener a las personas entretenidas no es la meta de la interpretación, sin embargo es necesario que así sea para mantener la atención de la audiencia en lo que se está presentando, durante el tiempo que dure la charla o recorrido.

b. Es pertinente. Para que la información se considere pertinente, debe cumplir con dos cualidades, una se refiere a que tiene un significado y la otra a que es personal. La información es significativa para nosotros cuando logramos relacionarla con algún conocimiento previo, es decir, la entendemos en el contexto de algo más que sabemos, y es personal cuando se relaciona lo que se está describiendo con algo dentro de la personalidad o experiencia del visitante.

c. Es organizada. La información es organizada, se debe presentar de una forma fácil de seguir, sin que sea necesario un gran esfuerzo por parte de la audiencia. Para ello se sugiere trabajar con cinco ideas principales o menos, de acuerdo con los estudios de (...) George Millar en 1956 sobre la capacidad de los seres humanos sobre cuanta información somos capaces de manejar.

Para hacer una interpretación organizada se debe de trabajar a partir de un tópico que es la idea principal y general que se desea transmitir al público. Y las distintas paradas de la interpretación ambiental se desarrollan a través de temas los cuales son escogidos 
según el tópico escogido y los elementos con los que se dispone para interpretar.

d. Tiene un tema. El tema es punto principal o mensaje que un comunicador está tratando de transmitir. En la interpretación, además del tema, hay un tópico, el cual es el objeto motivo de la presentación.

Los centros de interpretación tienen como objeto crear en quien acude a ellos una sensibilidad, conciencia, entendimiento, entusiasmo, compromiso, etc., hacia el recurso que es interpretado. Para cumplir la misión que tienen encomendada estos centros ; revelar al público los significados e interrelaciones del patrimonio natural y cultural- se cuenta con recursos expositivos e interpretativos clásicos, visitas guiadas y recorridos señalizados, además de otras actividades de sensibilización ambiental.

Los centros de interpretación son uno de los recursos de referencia para la transmisión de la cultura. Promueven el turismo y se conciben cada vez más como factores de valor económico, por su atractivo como actividad de ocio. Hacerlos más accesibles al público y conseguir generar experiencias atractivas para los visitantes, más allá de la mera observación, es el reto para una mejor difusión cultural.

\section{METODOLOGÍA}

Se ha empleado el método histórico, análisis y síntesis para dar a conocer la evolución de este tema, que brinda cultura, conocimiento, sobre los saberes ancestrales como patrimonio propio de un pueblo que no debe desconocer sus raíces, sus orígenes y en el ámbito educativo la investigación bibliográfica ha sido un soporte sustancial para este estudio.

\section{Criterios del propósito}

Es la representación tridimensional a escala de una obra compleja. En algunos casos la información que suministran los planos es insuficiente, por lo que se recurre a las maquetas. Su utilización desvela problemas constructivos que sólo con los planos no se habrían visto. En los planos, la visión espacial no es intuitiva, en las maquetas sí.

La interpretación pude utilizar muchas técnicas, pero también depende del arte de los que la realizan, haciendo notar que la clave está en el lenguaje que se utilice, en el dominio de las técnicas, pero sobretodo en el arte de conseguir llegar al corazón de las personas. Es importante considerar el cronograma de actividades que podría ser preparado para un período de cinco años, con actividades propuestas claramente para cada año. Una programación es importante por cuanto de esta manera el planificador puede observar el cronograma lógico de desarrollo de programas a corto plazo necesarios para llevar a cabo los planes de largo plazo.

Aunque la aparición de los potentes ordenadores o computadoras para diseño (CAD/CAM) ha sustituido en gran medida a las maquetas como herramientas de construcción, se siguen utilizando aún para dar una visión espacial de lo construido, mas no como ayuda durante la construcción. Es aconsejable que a los visitantes de un complejo turístico de cierta complejidad se les explique su funcionamiento utilizando una maqueta a escala, en la que, con facilidad y de una forma amena, se les van mostrando el plan masa en general, con sus distintas dependencias $y$ otros detalles de la instalación. La visión de la maqueta precede, normalmente, a la visita a las instalaciones.

El propósito de este análisis es describir las audiencias reales y potenciales para los programas interpretativos. Por tanto un programa interpretativo será de gran valor si es relacionado con los intereses, preferencias y necesidad del visitante. Por tanto, conociendo las características del visitante, los intérpretes pueden seleccionar mejor los métodos, programas, tiempos, tópicos y localizaciones más apropiados. La información relativa a las características del visitante puede ser obtenida a través de los datos existentes, técnicas de observación y encuestas.

- Perfil demográfico (procedencia, edad, nivel de educación)

- Perfil socio-económico (profesión u ocupación, ingresos, consumo de medios de comunicación mas utilizados)

- Información general del área a interpretar (principales fuentes de información acerca del lugar, evaluación de la veracidad y utilidad de información recibida a cada medio)

- Lugares de visita durante el viaje (otros lugares dentro del área a interpretar, motivaciones para visitar en el área, necesidades especiales) 
- Frecuencia de visita (modalidad de viajes, composición del grupo de viaje, permanencia en el área, gasto total durante su visita, intensión de retornar al lugar).

Criterio 1.- En las áreas protegidas existen dos tipos de letreros: administrativos (señales de tránsito y de orientación del visitante) e interpretativos. Como medio de interpretación, los letreros tienen varias ventajas ydesventajas: (...) Piray, M. (2008) Los letreros son susceptibles a daños y deterioro debido a la descomposición causada por condiciones climáticas (sol, humedad, arena y por el viento). Información impresa; libros, folletos, guías, mapas, carteles.

Criterio 2.- Aunque son considerados como medios, precisan de otros para llevarse a cabo. Se utilizan folletos, señales, paneles o grabaciones magnetofónicas, a través de un recorrido preestablecido. Incluyen películas, programas de diapositivas automáticas, postes de escucha, cintas grabadas transportables y todas las presentaciones audiovisuales no atendidas por personal.

Criterio 3.- Una exhibición es una de las formas más comunes de comunicación interpretativa. Es una colección de objetos o gráficas dispuestos de cierta forma para dar un mensaje a los visitantes. Los visitantes pueden observar las exhibiciones a su propio ritmo, generalmente sin guía alguna.

Desarrollo de actividades. Implica ejercitación de la habilidad por parte del público.

Ejemplos: el montar a caballo, escalar, la fotografía y cualquier otra actividad que lo involucre en una participación activa.

Criterio 4.- Generalmente, se utiliza a un experto ajeno al personal de plantilla, quien comenta o narra, en diversos estilos, sus experiencias, 0 traduce sus conocimientos a un lenguaje comprensible para el público.

Criterio 5.- Animación Pasiva.- El intérprete "interpreta" en forma teatral una actividad cotidiana, generalmente costumbrista, vistiendo como el personaje que representa. Se trata de revivir hechos y costumbres tradicionales, sin contar con la participación del público.

Animación activa.- Se refiere a los programas interpretativos con participación del público, en los que éste debe utilizar conocimientos previos o adquiridos durante la visita al área. Son las simulaciones, los juegos ambientales, las representaciones teatrales o la utilización de instrumentos con un fin más recreativo que el de adquisición dehabilidades.

\section{METODOLOGÍA PARA LA PREPARACIÓN DE PLANES DE INTERPRETACIÓN}

Análisis y síntesis de los antecedentes e información básica. Recopilación, revisión y análisis de información básica importante para la interpretación y educación ambiental, examinando la situación actual y proyecciones para elfuturo.

Las fuentes de colaboración y coordinación existentes y posibles para llevar a cabo programas de interpretación.

a) Identificar vacíos en la información y planificar inventario en el campo para obtener información necesaria.

b) Realizar inventario de campo sobre los aspectos mencionados y añadir esa información a lo recopilado, analizando lo en forma final.

c) Sintetizar y resumir lo sobresaliente en todos los aspectos.

- Elaborar las metas definitivas del plan

- Elaborar y analizar las limitaciones y condiciones para el proceso de planificación

- Elaborar una lista de limitaciones, dividida en las existentes que no se pueden modificar y las que se pueden modificar en algún grado.

- Elaborar una lista de las condiciones que favorecen la interpretación. 


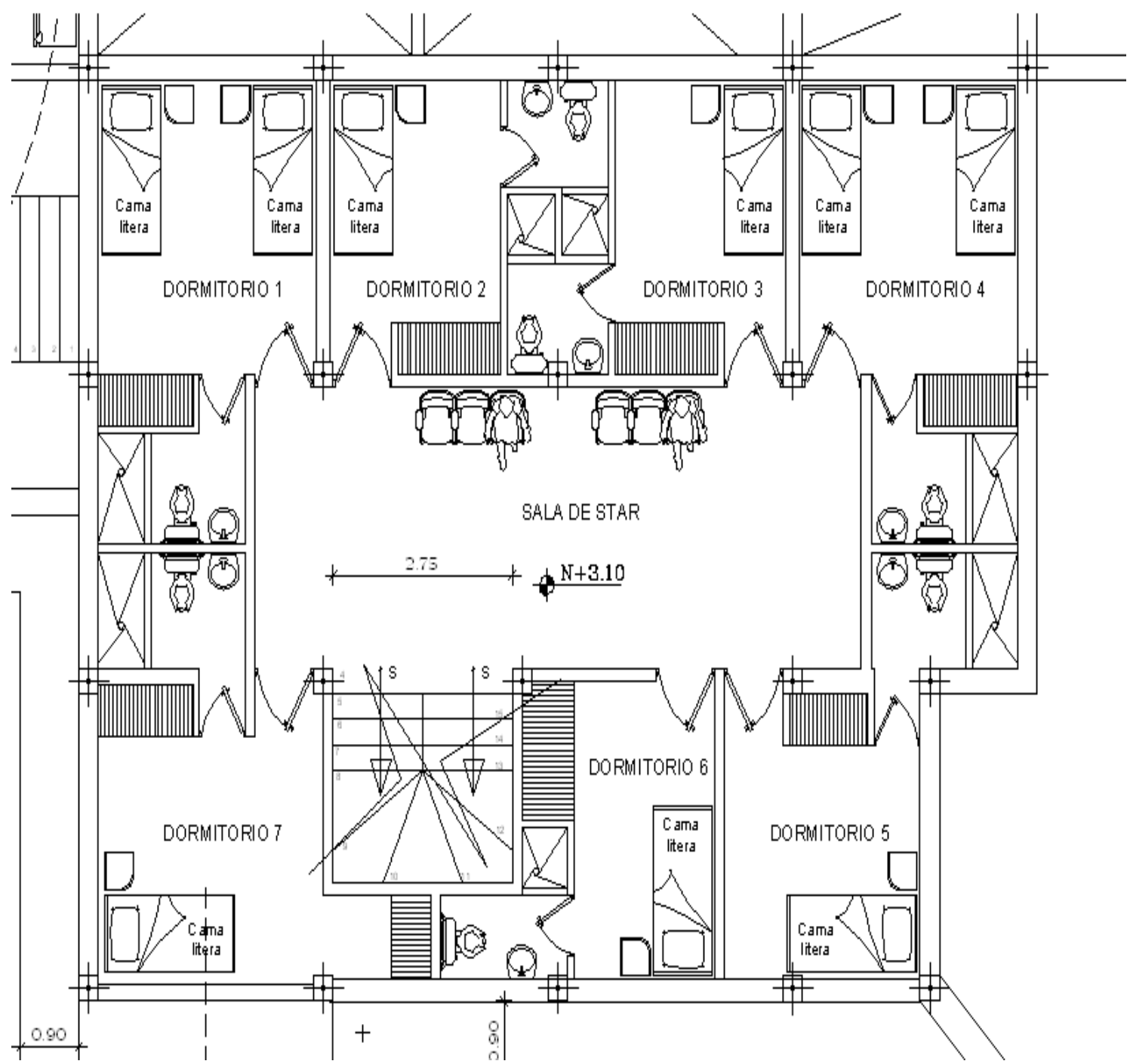

Planos Área de hospedaje especialmente para los Adultos Mayores:

Estas técnicas de uso muy extendido se utilizan cuando la inversión produce ingresos por sí misma, es decir, sería el caso de la tan mencionada situación de una empresa que vendiera servicios de informática.

El VPN y la TIR se aplican cuando hay ingresos, independientemente de que la entidad pague o no pague impuestos.

Las razones financieras son esenciales en el análisis financiero. Éstas resultan de establecer una relación numérica entre dos cantidades: las cantidades relacionadas corresponden a diferentes cuentas de los estados financieros de una empresa.
El análisis por razones o indicadores permite observar puntos fuertes o débiles de una empresa, indicando también probabilidades y tendencias, pudiendo así determinar qué cuentas de los estados financieros requiere de mayor atención en el análisis.

El adecuado análisis de estos indicadores permite encontrar información que no se encuentra en las cifras de los estados financieros. Las razones financieras por sí mismas no tienen mucho significado, por lo que deben ser comparadas con algo para poder determinar si indican situaciones favorables o desfavorables. 


\section{DATOS GENERALES}

001

1.1 Ficha №:

1.2 Evaluador: MSc. Consuelo Vergara / MSc. Yamil De Haz.

1.3 Fecha: 23/11/2016

\subsection{Nombre del Atractivo: CENTRO INTERPRETATIVO}

1.5 Categoría: Urbano-Rural.

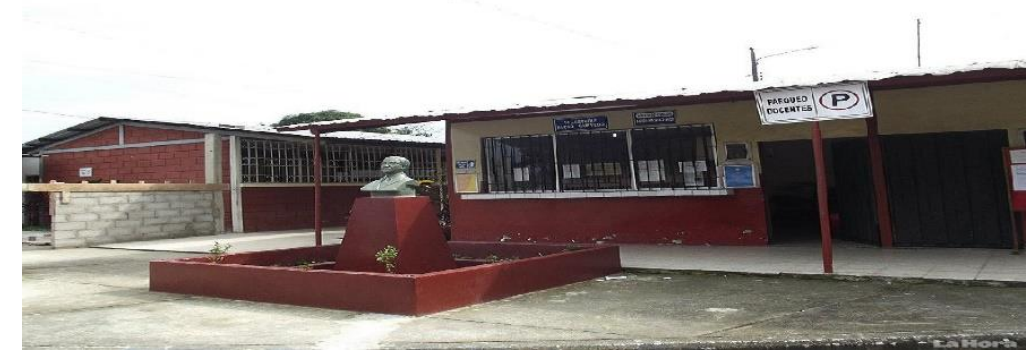

\section{UBICACIÓN}

\subsection{Provincia: Los Rios}

\subsection{Ciudad y/o Cantón: Quevedo.}

2.3 Localidad: Parroquia San Camilo

Se pueden encontrar árboles nativos como Guayacán, pachaco, balsa y otros introducidos como la teca, además en el sector aledaño ha sido desforestado por la existencia de los monos cultivos de la provincia.

El centro contará con animales propios del entorno natural del sector como: armadillos, ardillas, iguanas, lechuzas, guantas, guatusas, con piscina con peces como el boca chico propio de la zona, entre otros atractivos tanto de flora como de fauna.

Una casa de caña típica del sector con enseres campesino como muestra de las costumbres de la gente bella del campo.

Demostrar a la juventud a querer respetar sus raíces, y ese apego por el servicio comunitario y social para juntos hacer un país mejor. La casa de acogida para adultos mayores será un atractivo de esparcimiento.

\section{RESULTADOS}

Se ha mejorado de manera sustancial la educación poblacional y turística, como parte integral del desarrollo del Plan del Buen Vivir, considerando como parte integral de la sociedad las raíces culturales de nuestro origen sin causa alguna de negación.

A partir de los principales enfoques podemos observar que las posiciones extremas oscilan entre las que creen que es posible crecer físicamente y aquellas que consideran que el desarrollo sustentable tiene que ver más con la protección y conservación de la naturaleza. Cada uno de ellos es una expresión cerrada, de un punto de vista particular, de un grupo que enfoca "su verdad", pero que no toma en cuenta la verdad que encierran los puntos de vistas de los demás, las diversas interpretaciones se determinan por el contexto socioeconómico, político, científico e ideológico que rodea a quien expone su posición sobre la sustentabilidad. A pesar de su rápida aceptación y divulgación como expresión concentrada de un estilo de desarrollo cultural y ecológico, se destaca el carácter ambiguo de esta tesis, lo que ha condicionado la aparición de múltiples definiciones del desarrollo sustentable, en función de los intereses de los actores y de las circunstancias concretas del estudio del caso. 
1) Flujograma del proceso de recepción y bienvenida

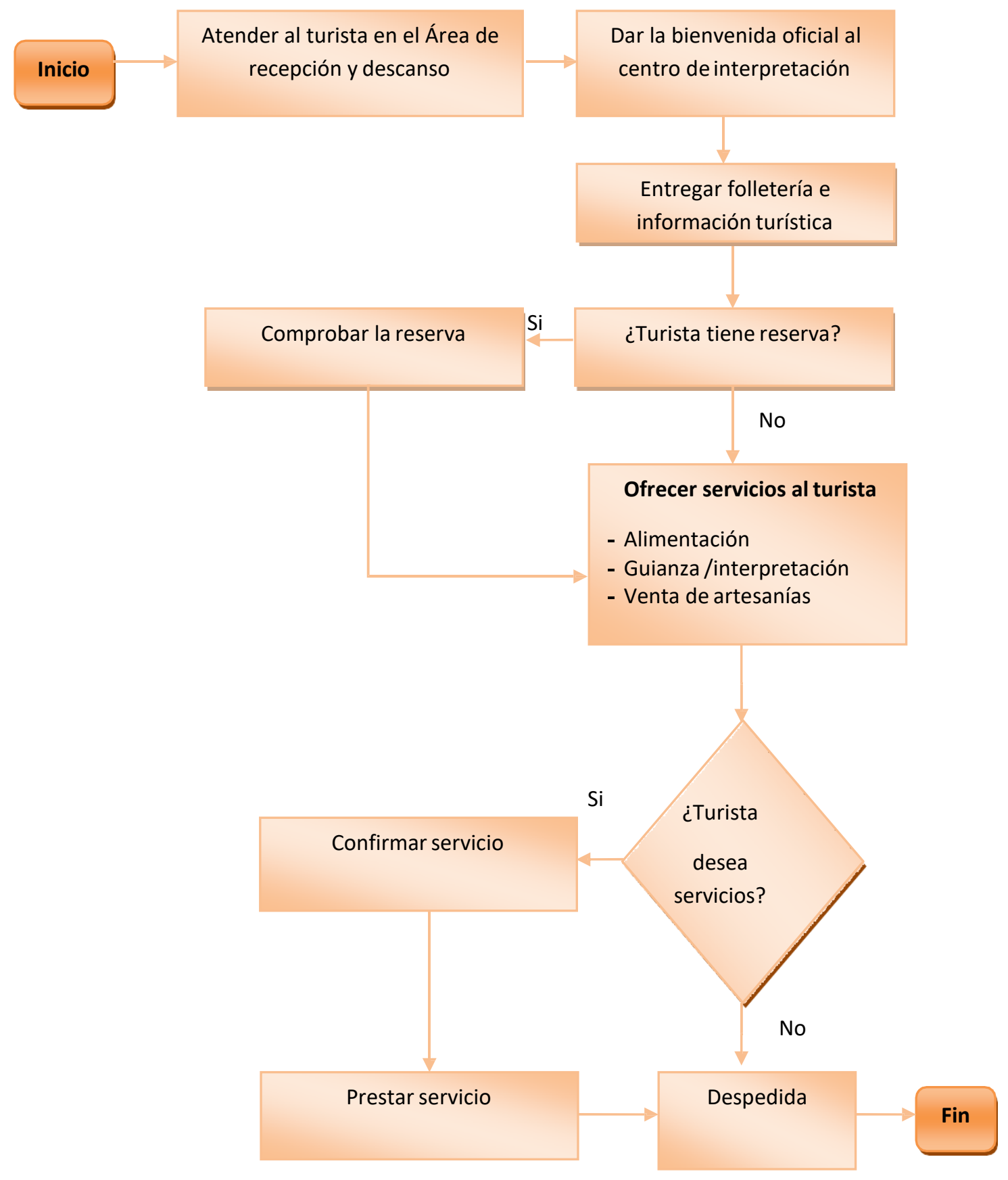


A pesar de dichas divergencias, que se mantienen hasta ahora, la discusión ha contribuido, al menos, a la aceptación de que el desarrollo sostenible y sustentable constituye un concepto multidimensional que involucra, como mínimo, dimensiones económicas, sociales y ambientales.

\section{Referencias Bibliográficas}

1. Dawson, L.. Cómo interpretar recursos naturales e históricos. Fondo Mundial para la Naturaleza (WWF). Turrialba, Costa Rica.130 p. 1999

2. Ecuador planeta mágico, Ecozoologico San Martín. "Aves". Consultado 20 de febrero 2010. Disponible en http://zoosanmartinaves.8m.com/zoo_a ves.htm

3. Escalona, I. Métodos de evaluación financiera en evaluación de proyectos. Consultado el 20 de septiembre 2008. Disponible en http:/www.monografías.com.
4. Grupo Técnico Espoch. Grupo de trabajo de la COCDIAG. 2008

5. Ibercaja. "Centro de Interpretación Ambiental" (en línea). Consultado 10 de septiembre 2009. Disponible en http://www.ibercaja.es.

6. Keith, Pennyfather. Interpretive and facilities. 1975.

7. Latorre, A,. "Manual de construcciones alternativas". Cap. I. 2008.

8. Tildel, Freeman. Interpreting Our Heritage. The University of North carolina Press, Chapel Hill. 1957.

9. Vargas, Enrique. Procesos de Aclimatación al Ambiente de Altura. 2005. La Paz- Bolivia. Disponible en http://www.ops.org.bo.

10. Wkipedia. La enciclopedia libre. "Análisis de audiencias". Consultado 15 de septiembre 2009. Disponible en http://es.wikipedia.org. 